\title{
BMJ Open Pre-emptive scalp infiltration with ropivacaine plus methylprednisolone versus ropivacaine alone for relief of postoperative pain after craniotomy in children (RP/MP vs RP): a study protocol for a randomised controlled trial
}

\author{
Chunmei Zhao, ${ }^{1}$ Yitong Jia, ${ }^{1}$ Zipu Jia, ${ }^{1}$ Xiong Xiao, ${ }^{2}$ Fang Luo ${ }^{3}$
}

\begin{abstract}
To cite: Zhao $\mathrm{C}$, Jia Y, Jia Z, et al. Pre-emptive scalp infiltration with ropivacaine plus methylprednisolone versus ropivacaine alone for relief of postoperative pain after craniotomy in children (RP/MP vs RP): a study protocol for a randomised controlled trial. BMJ Open 2019;9:e027864. doi:10.1136/ bmjopen-2018-027864

- Prepublication history and additional material for this paper are available online. To view these files, please visit the journal online (http://dx.doi. org/10.1136/bmjopen-2018027864).
\end{abstract}

Received 14 November 2018 Revised 17 April 2019 Accepted 21 May 2019

Check for updates

(C) Author(s) (or their employer(s)) 2019. Re-use permitted under CC BY-NC. No commercial re-use. See rights and permissions. Published by BMJ.

For numbered affiliations see end of article.

Correspondence to

Professor Fang Luo;

luofangwt@yahoo.com

\begin{abstract}
Introduction Pre-emptive scalp infiltration with local anaesthetics is the simplest and most effective method to prevent postoperative incisional pain. However, local infiltration of an anaesthetic only provides relatively short-term pain relief. Methylprednisolone (MP) treatment, administered as an adjuvant at the wound site, has been shown to provide satisfactory pain management after lumbar laminectomy. However, there is no evidence regarding the efficacy of MP infiltration for the relief of postoperative pain after craniotomy. Currently, postoperative pain after craniotomy in children is undertreated. Therefore, we aim to investigate whether pre-emptive scalp infiltration with ropivacaine (RP) plus $\mathrm{MP}$ is superior to RP alone to improve postoperative pain after craniotomy in children.
\end{abstract}

Methods and analysis The RP/MP versus RP trial is a prospective, single-centre, randomised, parallel-group study of 100 children aged 8-18 years undergoing intracranial surgery. Participants will be randomly allocated to receive pre-emptive scalp infiltration with either RP plus MP or RP alone. The primary outcome will be the cumulative fentanyl dose administered by patient-controlled intravenous analgesia within 24 hours postoperatively. The secondary outcomes will include postoperative Numerical Rating Scale scores, pain control satisfaction scores, length of stay and adverse events. Data will be analysed by the intention-to-treat principle. Ethical approval and dissemination The study protocol has been approved by the Institutional Review Board of Beijing Tiantan Hospital Affiliated to Capital Medical University (Approval Number: KY 2018-066-02). The results will be disseminated in international academic meetings and published in peer-reviewed journals. Trial registration number NCT03636165; Pre-results.

\section{BACKGROUND}

Until now, the idea that pain management following craniotomy is lacking in children

\section{Strengths and limitations of this study}

- Local infiltration only provides relatively short-term pain relief. The analgesic effects of a mixture of steroids and local anaesthetics may last longer. The ropivacaine (RP)/methylprednisolone (MP) versus RP trial is the first prospective randomised controlled study to confirm the analgesic effects of MP administered as an adjuvant as part of pre-emptive scalp infiltration in children undergoing craniotomy.

- Pre-emptive analgesia is defined as a preoperative treatment that is more effective than the same treatment postoperatively. The pre-emptive scalp infiltration blocks the introduction of nociceptive stimulation and inhibits the occurrence of central sensitisation, which provides a better analgesic effect than scalp infiltration before skin closure.

- The RP/MP versus RP trial will investigate whether pre-emptive scalp infiltration with RP plus only a single concentration of MP is superior to RP alone in improving postoperative pain after craniotomy in children. Further studies are needed to determine the optimal concentration of MP. Furthermore, because this trial is a single-centre study, it is necessary to perform multicentre clinical studies to provide higher levels of evidence.

has been rejected. The incidence of postoperative pain and the characteristics of pain after craniotomy in children are still rarely reported. Teo et al reported that $42 \%$ of children undergoing craniotomy had at least one episode of moderate or severe pain within 72 hours after the procedure. ${ }^{1}$ Pain is associated with adverse effects, such as hypertension and agitation, which may increase the risk of secondary intracranial haemorrhage, lead to a prolonged hospital stay and even 
increase mortality in children. ${ }^{2-4}$ However, postoperative pain in children has remained overlooked because of the difficulty of pain assessment in children and their limited expressive proficiency. ${ }^{5}$ If postoperative pain cannot be treated appropriately, children with recurrent pain are at a high risk of developing chronic pain after surgery. ${ }^{6}$ Therefore, adequate postoperative analgesia requires further study in children.

Currently, there are limited data describing pain management after neurosurgery in children. Children receiving multimodal analgesia, including strong opioids and non-steroidal anti-inflammatory drugs (NSAIDs), presented low pain intensity after major craniotomies. ${ }^{17}$ However, opioid-associated side effects, such as sedation and miosis, can mask early signs of neurological problems. There is also a potential risk of bleeding due to NSAIDs, particularly after craniotomy. Building an optimal pain management plan with a focus on reduction in opioids ${ }^{8}$ and NSAIDs is important.

Pain following craniotomy originates from the surgical incision in the pericranial muscles and soft tissues of the scalp. ${ }^{9}{ }^{10}$ Scalp infiltration with local anaesthetics is the simplest and most effective method of preventing postoperative incisional pain in paediatric patients. ${ }^{112}$ Cercueil et al reported that children younger than 2 years of age who received local anaesthetic infiltration had a decreased need for postoperative morphine. ${ }^{13}$ Local infiltration with a single anaesthetic only provides significant pain relief for a brief period, so many patients have significant pain after the local anaesthetics have dissipated. Many studies suggested that scalp infiltration could also improve postoperative pain in patients undergoing craniotomy, but only for the first few hours after surgery. ${ }^{14-18}$ Therefore, an optimal analgesic regimen to induce sufficient and prolonged analgesia is worth exploring to relieve pain in paediatric patients after major neurosurgical procedures.

Continuous incisional infiltration with local anaesthetics may be considered as a pain management method after knee and hip replacement due to improved analgesic efficacy. ${ }^{19}$ However, the major complications associated with this technique are mainly caused by catheter withdrawal, dislodgement or occlusion. In recent years, multiple modalities have been introduced to achieve adequate analgesia. The analgesic effects of a mixture of corticosteroids and local anaesthetics introduced into the articular cavity have been reported to be sustained for 3 days after total knee arthroplasty (TKA).$^{20}$ Meanwhile, according to the American College of Rheumatology, intra-articular (IA) corticosteroid injections may be recommended in people with knee osteoarthritis to provide a short-term reduction in moderate to severe pain. ${ }^{21}{ }^{22}$ Therefore, corticosteroids administered as an adjuvant may be a good way to prolong the analgesic time of local incisional infiltration.

Methylprednisolone (MP) is a type of glucocorticoid with potent anti-inflammatory effects and a high affinity for its receptor. The topical application of MP has been demonstrated to suppress transmission via $\mathrm{C}$-fibres but not via A-beta fibres through direct membrane action. ${ }^{23}$ Gurava Reddy et alsuggested that the addition of MP to an injectable analgesic cocktail infiltration for periarticular infiltration in patients undergoing unilateral TKA leads to better postoperative analgesia in the early postoperative period of 24-48 hours. ${ }^{24}$ Administration of a single IA multidrug, including MP, RP and epinephrine, on the first day following TKA may provide better pain relief during the 5 days following surgery. ${ }^{25} \mathrm{MP}$ administered only at the incision site instead of systemically achieved satisfactory pain management at the end of 24 hours in adults undergoing lumbar laminectomy. ${ }^{26}$ The local application of MP, with a strong analgesic effect, may further improve the analgesic effect of scalp infiltration. However, there is no evidence regarding the efficacy of pre-emptive scalp infiltration with RP plus MP for relief of postoperative pain after craniotomy in children.

Based on the current literature, we hypothesise that, compared with RP alone, RP plus MP will provide improved postoperative pain management after craniotomy in children aged 8-18 years, and we will perform the following randomised controlled trial.

\section{METHODS AND ANALYSIS \\ Trial design}

The RP/MP versus RP trial is a single-centre, randomised, parallel-group clinical trial of patients scheduled to undergo elective craniotomies and receive pre-emptive scalp infiltration with either RP plus MP or RP alone in a 1:1 group ratio for the two study arms (figure 1 ). The trial will be conducted at Beijing Tiantan Hospital Affiliated with Capital Medical University. This study, which will begin in December 2018, will have a duration of 1 year.

\section{Objectives}

The aim of the RP/MP versus RP trial is to determine whether MP plus RP is superior to RP alone in relieving postoperative pain in children undergoing intracranial surgery.

\section{Eligibility criteria}

Patients must meet all of the following criteria to be eligible:

1. Age $8-18$ years.

2. Elective craniotomy under general anaesthesia.

3. American Society of Anaesthesiologists (ASA) physical status of I or II.

4. Anticipated full recovery within 2 hours postoperatively.

5. Informed consent provided by the patient's parent(s) and/or legal guardian.

Subjects with one or more of the following conditions will be excluded:

1. History of allergies to any of the study drugs.

2. Chronic opioid use (3 days per week for more than 1 month), use of any painkiller within 24 hours before surgery and steroid use. 


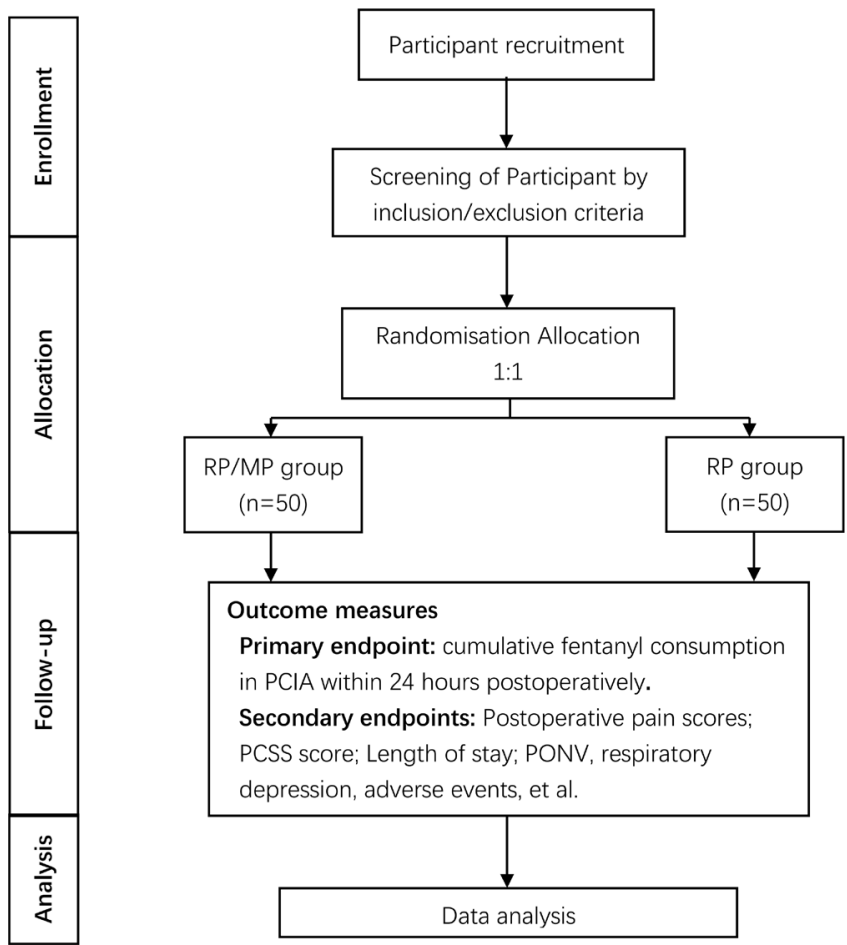

Figure 1 Study flow diagram of the RP/MP versus RP trial. MP, methylprednisolone; PCIA, patient-controlled intravenous analgesia; PCSS, pain control satisfaction score; PONV, postoperative nausea and vomiting; RP, ropivacaine.

3. Psychiatric disorders.

4. Uncontrolled epilepsy.

5. Chronic headache.

6. Peri-incisional infection.

7. Body mass index exceeding the 99th percentile for age.

8. Children who cannot use patient-controlled intravenous analgesia (PCIA) devices.

9. Children who cannot understand the instructions for the pain scales before surgery.

\section{Allocation and blinding}

The participants will be randomised into two treatment arms: an experimental group (RP/MP treatment group) and a control group (RP treatment group). The subjects will be allocated to the two arms in a 1:1 ratio using simple randomisation. The randomisation will be organised by the pharmacy department using a computerised randomisation table. The random numbers will be kept in opaque sealed envelopes and opened by an independent anaesthesiologist who will not be involved in the study, and he or she will be responsible for preparing the study drugs in identical syringes labelled only with the drug's serial number. Then, the study drugs will be delivered to the study surgeon performing scalp infiltration. During the study period, the patients and outcome assessors will be blinded to the group randomisation. All other aspects of the perioperative pain protocol will be identical between the two groups.

\section{Interventions}

All included patients will be allocated to one of the following two study groups. Patients in the RP/MP group will receive peri-incisional scalp infiltration with miscible liquids consisting of $0.125 \% \mathrm{MP}, 0.2 \% \mathrm{RP}$ and normal saline miscible liquids. ${ }^{27}$ Patients in the RP group will receive peri-incisional scalp infiltration with miscible liquids consisting of $0.2 \% \mathrm{RP}$ and normal saline. The infiltration solution will be prepared by the first anaesthesiologist in a separate operating room. The assigned solution will be injected subcutaneously by the surgeon along the incision and throughout the entire thickness of the scalp before a skin incision is performed. The volume of local infiltration solution will be determined by the surgeon according to the incision length, and the amount of solution used will be recorded by the investigators. The study drug will be supplied as a syringe of liquid, identical in volume but indicated by a letter, for both groups. For the scalp infiltrations, a straight $23 \mathrm{G}$ needle will be used.

\section{Anaesthesia induction and management}

In the ward, all children will receive midazolam $0.5 \mathrm{mg}$ / $\mathrm{kg}$ orally $20 \mathrm{~min}$ before being transferred to the operating room. On arrival to the operating room, standard monitoring will be established (pulse oximetry, electrocardiography and non-invasive arterial blood pressure monitoring). Peripheral vascular access will be established while the children are breathing $50 \%$ nitrous oxide in oxygen. Anaesthesia will be induced with $2 \mathrm{mg} /$ $\mathrm{kg}$ propofol, $0.6 \mathrm{mg} / \mathrm{kg}$ rocuronium and $3 \mu \mathrm{g} / \mathrm{kg}$ fentanyl after obtaining intravenous access.

After intubation, anaesthesia will be maintained with sevoflurane $(1.5 \%)$ and remifentanil $(0.17 \mu \mathrm{g} / \mathrm{kg} / \mathrm{h})$. Ventilation with $60 \%$ oxygen and $40 \%$ air will be controlled to maintain an end-tidal carbon dioxide (PETCO2) of $30-40 \mathrm{mmHg}$. Before proceeding to surgery, the children will receive peri-incisional scalp infiltration performed by the surgeon according to their group allocation. Five minutes after local infiltration, surgery will be performed by the same surgeon. The mean arterial pressure (MAP) and heart rate (HR) will be maintained within $30 \%$ of the baseline values with vasoactive drugs. Fentanyl will be administered to attenuate potent stress responses induced by noxious stimuli at certain time points, such as scalp incision or skull drilling. No acetaminophen or other additional analgesics will be administered. The dosage of all drugs and intraoperative physical parameters will be closely monitored and recorded by the investigator.

After surgery, atropine $0.01 \mathrm{mg} / \mathrm{kg}$ and neostigmine $0.05 \mathrm{mg} / \mathrm{kg}$ will be administered intravenously to antagonise any residual neuromuscular blockade. Extubation will be performed after confirmation of eye opening, adequate spontaneous respiration and purposeful movements. Additionally, all patients will receive tropisetron hydrochloride $(0.2 \mathrm{mg} / \mathrm{kg}$ to a maximum dose of $5 \mathrm{mg})$ intravenously.

After extubation, patients will be transferred to the postoperative care unit (PACU). The postoperative 
PCIA formulation will be set as fentanyl $(20 \mu \mathrm{g} / \mathrm{kg})$ and tropisetron hydrochloride $(0.2 \mathrm{mg} / \mathrm{kg})$ diluted to a total volume of $100 \mathrm{~mL}$ in normal saline. The PCIA device will provide a bolus $(0.5 \mu \mathrm{g} / \mathrm{kg}, 10 \mathrm{~min}$ lock-out time $)$, and the maximum dose will be limited to $2 \mu \mathrm{g} / \mathrm{kg}$ per hour. ${ }^{28-30}$ If the patient experiences inadequate analgesia four times after the fentanyl bolus, the bolus dose will be increased to $1 \mu \mathrm{g} / \mathrm{kg}$, and the maximum dose will be increased to $4 \mu \mathrm{g} / \mathrm{kg}$ per hour. Both the initial dose and background infusion of the PCIA in this study will be set at 0 . The button on the analgesic pump will be pressed when the patient reports an Numerical Rating Scale (NRS) score of 4 or more or at the request of the patient or the parent. The modified Aldrete score will be used as an objective assessment tool to guide discharge from the PACU to a ward. A score $>9$ will be required for discharge. ${ }^{31}$ The MAP, HR and SpO2 will be monitored for 4 hours in the ward. If vomiting occurs more than twice in $2 \mathrm{~min}$, metoclopramide $(2.5-5 \mathrm{mg})$ will be intravenously injected.

\section{Outcomes}

Table 1 provides an overview of the outcomes and assessment time points.

\section{Primary outcome}

The primary outcome will be the cumulative fentanyl consumption administered via the PCIA device within 24 hours postoperatively.

\section{Secondary outcomes}

Secondary outcomes will include the following aspects:

- Postoperative analgesia: The number of participants who receive no fentanyl, the first time the patient presses the PCIA button, which will be defined as the time from the beginning of the postoperative period to the first administration of analgesics and the total number of times that the patient presses the PCIA button, including effective presses and ineffective presses, will be recorded.

- Postoperative pain scores: A NRS will be administered to assess postoperative pain intensity by a research assistant who will receive specific training regarding the use of this scale and will be blinded to the grouping. The NRS score ( $0=$ no pain; $10=$ the worst possible pain) will be assessed at fixed intervals after the procedure, that is, 2 hours, 4 hours, 8 hours, 24 hours, 48 hours, 72 hours, 1 week, 2 weeks, 1 month, 3 months and 6 months after surgery. Significant or moderate pain will be defined as an NRS score $\geq 4$. Severe pain will be defined as a pain score $\geq 7$.

- Postoperative recovery: Patient satisfaction will be assessed by the pain control satisfaction score (PCSS) (0 for unsatisfactory and 10 for very satisfactory) at 24 hours, 48 hours, 72 hours, 1 week, 1 month, 3 months and 6 months after surgery. The length of stay (LOS) will be recorded as the number of nights spent in the hospital after surgery. The times to first water consumption and oral intake request and scales of oral intake after surgery will also be recorded.

- Adverse events (AEs): Postoperative nausea and vomiting, respiratory depression, pruritus, hypotension, bradycardia, emergence delirium, oversedation and any signs of local anaesthetic or steroid-associated systemic toxicity within 24 hours postoperatively will be recorded. Respiratory depression will be defined as persistent (more than $1 \mathrm{~min}$ ) oxygen desaturation of $90 \%$, a respiratory rate of less than 8 breaths $\mathrm{min}^{-1}$ or oxygen desaturation of less than $94 \%$ along with a respiratory rate less than 10 breaths $\mathrm{min}^{-1}$ requiring supplemental oxygen to maintain $\mathrm{SpO} 2$ at more than $94 \%$ in the absence of clinically obvious upper airway obstruction. ${ }^{32}$ Emergence delirium will be assessed using the Paediatric Anaesthesia Emergence Delirium scale. ${ }^{33}$ Emergence agitation will be considered to correspond to a total score of $>12$ at any time. Sedation will be assessed using the Ramsay Sedation Scale. ${ }^{34}$

- Vital signs: HR and MAP will be recorded before anaesthetic induction, after anaesthetic induction, after scalp infiltration, during skull drilling, during dura mater cutting and during skin closure at 2 hours, 4 hours, 8 hours and 24 hours after surgery.

- Incision-related AEs: Related AEs will be assessed, including delayed incisional healing, incisional infection, intracranial infection and scar healing after surgery. The wounds will be assessed using the Wound Healing Score, including skin healing, infection and hair regrowth at 3 and 6 weeks postoperatively. ${ }^{35}$ To quantify scar healing, Patient and Observer Scar Assessment Scale scores will be assessed at 6 months postoperatively. ${ }^{36}$ In addition, the time of removal of stitches or surgical drains will also be recorded.

- Occurrence of AEs and serious adverse events (SAEs): An AE will be defined as any untoward medical occurrence. An SAE will include death, immediately life-threatening conditions, coma, inpatient hospitalisation or prolongation of existing hospitalisation. AEs and SAEs will be observed throughout the study.

\section{Safety}

All AEs for which the investigator or the sponsor determines to have a causal relationship with the investigational treatment during the trial will be recorded. An SAE between study enrolment and hospital discharge will be reported to the trial management committee within 24 hours. An SAE report will note the date of occurrence, severity, intensity, relationship with the treatment (or the study), evaluation and outcome. All SAEs will be addressed to resolution or stabilisation. If the patients are harmed as a result of their participation in the trial, they will receive free treatment and adequate compensation. All patients will be under close personal supervision by the outcome assessors until their follow-up visit 6 months after surgery. The chief investigator will be responsible for all AEs reported. 


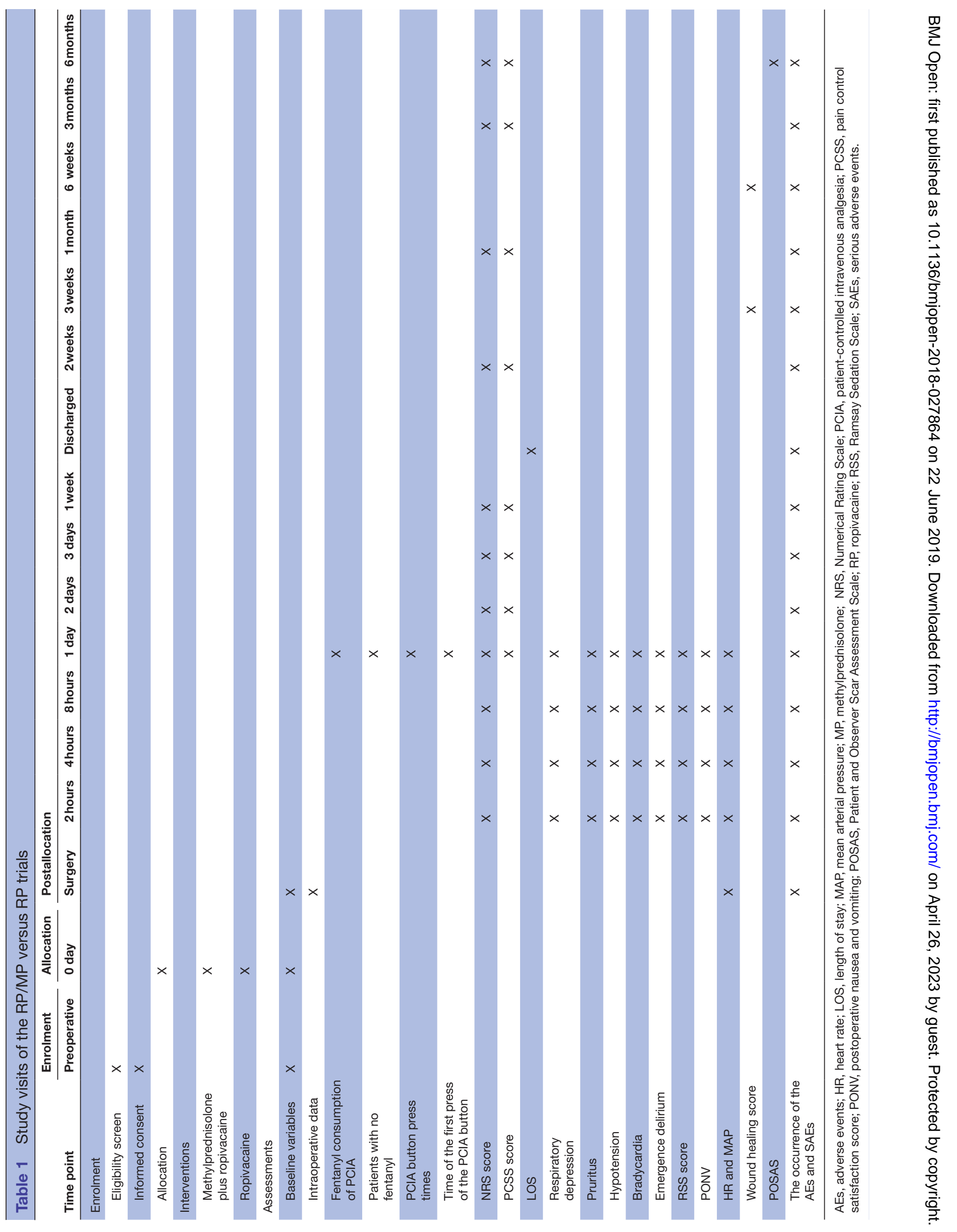




\section{Missing values}

Missing data will not be replaced. Mixed models will be used in the analysis of repeated data to avoid deleting subjects with any missing values.

\section{Sample size calculation}

According to Ju's article, the total fentanyl consumption during the first 24hours after surgery was 103.9 (11.5) $\mu \mathrm{g}$ for children who received local infiltration with RP for tonsillectomy and adenoidectomy. ${ }^{37}$ Maxwell et al reported that paediatric craniotomy patients receive approximately $300 \mu \mathrm{g} / \mathrm{kg}$ of morphine on the first postoperative day. ${ }^{38}$ This dose of morphine is converted to an equianalgesic dose of fentanyl (the conversion factor is $1 \mathrm{mg}$ morphine $=10 \mu \mathrm{g}$ fentanyl), that is, paediatric craniotomy patients receive approximately $3 \mu \mathrm{g} / \mathrm{kg}$ of fentanyl on the first postoperative day. Combined with the previous experience of our centre, we hypothesise that the total fentanyl consumption during the first 24hours after craniotomy will be $110 \pm 30 \mu \mathrm{g}$ for children who received local infiltration with RP.

Although Ersayli's study did not identify any extra effect of MP in patients undergoing lumbar discectomy, ${ }^{27}$ this study suggested a trend that local infiltration by MP could reduce the amount of postoperative analgesics in the 24 hours after lumbar discectomy by $20 \%$. We hypothesise that the local application of MP will reduce the dosage of fentanyl by $20 \%$, that is, $88 \pm 30 \mu \mathrm{g}$, within 24 hours after paediatric craniotomy.

Therefore, we estimate that the accumulated fentanyl dose after 24 hours will be approximately $110 \pm 30 \mu \mathrm{g}$ in the RP group and $88 \pm 30 \mu \mathrm{g}$ in the RP/MP group. Forty patients per group will be needed based on a $90 \%$ power with a two-sided $\alpha$ level of 0.05 to show a relative betweengroup difference of $20 \%$ in the composite primary outcome measure. Considering a dropout rate of $20 \%$, a total of 50 patients per arm will be required; thus, the total sample size will be 100 patients in this RP/MP versus RP trial.

\section{Statistical analysis}

For descriptive variables, we plan to use the mean and SD to report normally distributed variables and the median and IQR for skewed distributions. Dichotomous and categorical data will be described as the number of patients/ episode and percentages. The Kolmogorov-Smirnov $\mathrm{Z}$ test will be used to analyse the equality of the distributive functions of the variables.

Endpoint data will be analysed according to the intention-to-treat principle, and all participants will be analysed once enrolled. Baseline characteristics of the children will be compared between groups using the non-parametric Mann-Whitney/Wilcoxon test for continuous data or the $\chi^{2}$ or Fisher's exact test for categorical data. For numerical data collected more than once (eg, NRS, PCSS, haemodynamic parameters and Wound Healing Score) during the study period, two-way repeated measures analysis of variance on ranks will be performed with time of measurement as the repeated factor and group as the non-repeated factor. Potential risk factors (eg, age and sex) for moderate or severe postoperative pain after surgery will be identified using univariate analyses. For dichotomous pain descriptors (moderate to severe pain and severe pain), multivariate analysis will be used to determine possible confounding covariates, such as age, body mass index and type of surgery. The results of the regression analyses will be presented as ORs and 95\% CIs. Posthoc comparisons will include within-group changes over time and between-group differences at each assessment. A $p<0.05$ will be considered statistically significant. Statistical analyses will be performed with SPSS software, V.23.

\section{DISCUSSION}

The RP/MP versus RP trial will be the first rigorous trial to examine the analgesic effects of MP administered as an adjuvant as part of pre-emptive scalp infiltration in children undergoing craniotomy and may provide guidance for paediatric postoperative pain management. We plan to recruit children aged 8-18 years old for the following reasons. Several studies have noted that older children experience more severe postoperative pain than younger children and that pain intensity increases with age..$^{39-41}$ Sowder et al found that children with an average age of 5 years tended to receive ibuprofen alone after tonsillectomy; however, older patients with a mean age of 8 years were more likely to receive potent opioids or ibuprofen combined with anaesthetics. ${ }^{42}$ Older children may need more painkillers than younger children because they have different pain thresholds. Berde et al also noted that it seems reasonable for children aged over 7 years to receive PCIA because they are at a developmentally mature age ${ }^{43}$ Consequently, combined with the characteristics of paediatric pain and postoperative analgesia, we will select school-aged children and adolescents who are able to learn how to use PCIA devices and will not result in deviations from the endpoint due to age.

In this study, $0.2 \% \mathrm{RP}$ will be administered, and this concentration is generally recommended by the European Society for Paediatric Anaesthesiology (ESPA) Pain Committee ${ }^{29}$ In children, $0.025 \%$ dexamethasone can be applied locally, which is the most frequently used concentration in clinical practice and provides effective postoperative analgesia during otolaryngology and orthopaedic surgery. ${ }^{37} 44$ The potency of dexamethasone, however, is five times stronger than that of MP. ${ }^{45}$ The calculated concentration of topical MP used in this study is $0.125 \%$, and this concentration is similar to that used in adults. ${ }^{27}$ There is no significant difference in the concentration of topical glucocorticoids used between children and adults, and the amount injected may vary depending on weight. Therefore, $0.125 \% \mathrm{MP}$ will be used in this study, as this dose is safe and effective for children.

This study has several limitations. First, we will use single doses of MP as an adjuvant to local anaesthetics, 
which are often used and identified in clinical practice. Further studies with different local doses of methylprednisolone as an infiltration adjuvant may be warranted to determine the optimal topical concentration to achieve the best analgesic effect and minimise the risk of side effects. In addition, this study is also a single-centre trial. Therefore, it is necessary to perform multicentre clinical studies with a larger sample size to provide higher levels of evidence.

In summary, the results from the RP/MP versus RP trial may provide an effective method to alleviate postoperative pain after craniotomy in older children and provide a significant contribution to the development of paediatric multimodal analgesia.

\section{ETHICS AND DISSEMINATION}

\section{Ethical and legislative approvals}

The RP/MP versus RP trial is registered at ClinicalTrials. gov with the trial identification number NCT 03636165. The trial methods and results will be reported according to the Consolidated Standards of Reporting Trials (CONSORT) 2010 guidelines. ${ }^{46}$ Amendments to the protocol will only be made by an academic committee and approved by the Institutional Review Board at Beijing Tiantan Hospital Affiliated to Capital Medical University. Signed informed consent will be obtained from the patient's parent(s) and/or legal guardian (see online supplementary file 1). If a child is able to express himself or herself in writing, the child will be invited to sign a simplified child assent form (see online supplementary file 2) appropriate to his or her capacity for expression. All changes will be recorded. Any change will be applied to all subsequent patients, and the registration record will be updated.

\section{Publication plan}

Scientific statements and reports corresponding to the study will be written under the responsibility of the coordinating investigator of the study, with the consent of the principal investigators and the methodologist. The co-authors of the report and the publications will be the investigators and clinicians involved, on a pro rata basis of their contribution in the study, as well as the biostatistician and associated researchers. Rules regarding publication will follow international recommendations, and publications will be submitted to peer-reviewed journals. ${ }^{47}$

\section{Author affiliations \\ ${ }^{1}$ Department of Anesthesiology, Beijing Tiantan Hospital, Capital Medical University, Beijing, China \\ ${ }^{2}$ Department of Neurosurgery, Beijing Tiantan Hospital, Capital Medical University, Beijing, China \\ ${ }^{3}$ Department of Pain Management, Beijing Tiantan Hospital, Capital Medical University, Beijing, China}

Contributors $\mathrm{CZ}$ and $\mathrm{YJ}$ contributed equally to this work and should be considered co-first authors. CZ and YJ contributed to the conception and drafting of the first manuscript for this trial. FL is the principal investigator of the entire study and edited the final manuscript. ZJ and XX contributed to the conception of the research protocol and will participate in the follow-up for this trial. All authors critically revised and modified the protocol and the article. They all approved the final version to be published.

Funding This study was funded by Beijing Municipal Administration of Hospitals Clinical Medicine Development of Special Funding Support (No. XMLX201707).

Competing interests None declared.

Patient consent for publication Obtained.

Provenance and peer review Not commissioned; externally peer reviewed.

Data sharing statement Substantive protocol amendments will be reported, reviewed and approved by the local medical ethical committee before application. The frequency of monitoring visits will be determined by the site enrolment rate. Rules on publication will follow international recommendations.

Open access This is an open access article distributed in accordance with the Creative Commons Attribution Non Commercial (CC BY-NC 4.0) license, which permits others to distribute, remix, adapt, build upon this work non-commercially, and license their derivative works on different terms, provided the original work is properly cited, appropriate credit is given, any changes made indicated, and the use is non-commercial. See: http://creativecommons.org/licenses/by-nc/4.0/.

\section{REFERENCES}

1. Teo J, Palmer G, Davidson A, et al. Post-craniotomy pain in a paediatric population. Anaesthesia 2011;39:89.

2. Basali A, Mascha EJ, Kalfas I, et al. Relation between perioperative hypertension and intracranial hemorrhage after craniotomy. Anesthesiology 2000;93:48-54.

3. Kim JS, Kim GW, Park DH, et al. Effects of scalp nerve block on pain and emergence agitation after paediatric nevus surgery: a clinical trial. Acta Anaesthesiol Scand 2017;61:935-41.

4. Kulshrestha A, Bajwa SJS. Management of acute postoperative pain in pediatric patients. Anaesthesia, Pain Intensive Care Medicine 2014;18.

5. Jimenez N, Jackson DL, Zhou C, et al. Postoperative pain management in children, parental English proficiency, and access to interpretation. Hosp Pediatr 2014;4:23-30.

6. Nikolajsen L, Brix LD. Chronic pain after surgery in children. Curr Opin Anaesthesiol 2014;27:507-12.

7. Bauer DF, Waters AM, Tubbs RS, et al. Safety and utility of scheduled nonnarcotic analgesic medications in children undergoing craniotomy for brain tumor. Neurosurgery 2010;67:353-6. Discussion 55-6.

8. Ferland CE, Vega E, Ingelmo PM. Acute pain management in children: challenges and recent improvements. Curr Opin Anaesthesiol 2018;31:327-32.

9. Puntis M, Garner A. Management of pain following craniotomy. Br J Nurs 2015;24:740-4.

10. Haldar R, Kaushal A, Gupta D, et al. Pain following craniotomy: reassessment of the available options. Biomed Res Int 2015;2015:1-8.

11. Song J, Li L, Yu P, et al. Preemptive scalp infiltration with $0.5 \%$ ropivacaine and $1 \%$ lidocaine reduces postoperative pain after craniotomy. Acta Neurochir 2015;157:993-8.

12. Chaki T, Sugino S, Janicki PK, et al. Efficacy and safety of a lidocaine and ropivacaine mixture for scalp nerve block and local infiltration anesthesia in patients undergoing awake craniotomy. J Neurosurg Anesthesiol 2016;28:1-5.

13. Cercueil E, Migeon A, Desgranges FP, et al. Postoperative analgesia for craniosynostosis reconstruction: Scalp nerve block or local anesthetic infiltration? Paediatr Anaesth 2018;28:474-5.

14. Hansen MS, Brennum J, Moltke FB, et al. Pain treatment after craniotomy: where is the (procedure-specific) evidence? A qualitative systematic review. Eur J Anaesthesiol 2011;28:821-9.

15. Law-Koune JD, Szekely B, Fermanian C, et al. Scalp infiltration with bupivacaine plus epinephrine or plain ropivacaine reduces postoperative pain after supratentorial craniotomy. J Neurosurg Anesthesiol 2005;17:139-43.

16. Saringcarinkul A, Boonsri S. Effect of scalp infiltration on postoperative pain relief in elective supratentorial craniotomy with $0.5 \%$ bupivacaine with adrenaline 1:400,000. J Med Assoc Thai 2008;91:1518-23.

17. Biswas BK, Bithal PK. Preincision $0.25 \%$ bupivacaine scalp infiltration and postcraniotomy pain: a randomized double-blind, placebo-controlled study. J Neurosurg Anesthesiol 2003;15:234-9.

18. Batoz $\mathrm{H}$, Verdonck $\mathrm{O}$, Pellerin $\mathrm{C}$, et al. The analgesic properties of scalp infiltrations with ropivacaine after intracranial tumoral resection. Anesth Analg 2009;109:240-4. 
19. Liu SS, Richman JM, Thirlby RC, et al. Efficacy of continuous wound catheters delivering local anesthetic for postoperative analgesia: a quantitative and qualitative systematic review of randomized controlled trials. J Am Coll Surg 2006;203:914-32.

20. Ikeuchi M, Kamimoto Y, Izumi M, et al. Effects of dexamethasone on local infiltration analgesia in total knee arthroplasty: a randomized controlled trial. Knee Surg Sports Traumatol Arthrosc 2014;22:1638-43.

21. Hochberg MC, Altman RD, April KT, et al. American College of Rheumatology 2012 recommendations for the use of nonpharmacologic and pharmacologic therapies in osteoarthritis of the hand, hip, and knee. Arthritis Care Res 2012;64:465-74.

22. Di Sante L, Venditto T, loppolo F, et al. Ultrasound guided injection of a painful knee osteoarthritis with medial meniscus extrusion: a case series study. Muscles Ligaments Tendons J 2017;7:331-7.

23. Johansson A, Hao J, Sjölund B. Local corticosteroid application blocks transmission in normal nociceptive C-fibres. Acta Anaesthesiol Scand 1990;34:335-8.

24. Gurava Reddy AV, Thayi C, Natarajan N, et al. Validating the role of steroid in analgesic cocktail preparation for local infiltration in total knee arthroplasty: a comparative study. Anesth Essays Res 2018;12:903-6.

25. Iseki T, Tsukada S, Wakui M, et al. Percutaneous periarticular multi-drug injection at one day after total knee arthroplasty as a component of multimodal pain management: a randomized control trial. BMC Musculoskelet Disord 2019;20:61.

26. Gurbet A, Bekar A, Bilgin $\mathrm{H}$, et al. Preemptive wound infiltration in lumbar laminectomy for postoperative pain: comparison of bupivacaine and levobupivacaine. Turk Neurosurg 2014;24:48-53.

27. Ersayli DT, Gurbet A, Bekar A, et al. Effects of perioperatively administered bupivacaine and bupivacaine-methylprednisolone on pain after lumbar discectomy. Spine 2006;31:2221-6.

28. Hurley RW, Murphy JD, CI WU, et al. In: Miller RD, ed. Miller's Anesthesia. 8 ed: Elsevier 2015.

29. Vittinghoff M, Lönnqvist PA, Mossetti V, et al. Postoperative pain management in children: Guidance from the pain committee of the European Society for Paediatric Anaesthesiology (ESPA Pain Management Ladder Initiative). Paediatr Anaesth 2018;28:493-506.

30. Anghelescu DL, Faughnan LG, Oakes LL, et al. Parent-controlled PCA for pain management in pediatric oncology: is it safe? $\mathrm{J}$ Pediatr Hematol Oncol 2012;34:416-20.

31. Abdullah HR, Chung F. Postoperative issues: discharge criteria. Anesthesiol Clin 2014;32:487-93.

32. Sadhasivam S, Chidambaran V, Zhang X, et al. Opioid-induced respiratory depression: ABCB1 transporter pharmacogenetics. Pharmacogenomics J 2015;15:119-26.

33. Sikich N, Lerman J. Development and psychometric evaluation of the pediatric anesthesia emergence delirium scale. Anesthesiology 2004;100:1138-45.
34. Sessler CN, Grap MJ, Ramsay MA. Evaluating and monitoring analgesia and sedation in the intensive care unit. Crit Care 2008;12(Suppl 3):S2.

35. Langford P, Wolfe R, Danks RA. Wound healing after craniotomy: a randomized trial comparing scalp clips to artery forceps for scalp hemostasis. J Neurosurg 2009;111:1175-8.

36. Fearmonti R, Bond J, Erdmann D, et al. A review of scar scales and scar measuring devices. Eplasty 2010;10:e43.

37. Ju NY, Cui GX, Gao W. Ropivacaine plus dexamethasone infiltration reduces postoperative pain after tonsillectomy and adenoidectomy. Int J Pediatr Otorhinolaryngol 2013;77:1881-5.

38. Maxwell LG, Buckley GM, Kudchadkar SR, et al. Pain management following major intracranial surgery in pediatric patients: a prospective cohort study in three academic children's hospitals. Paediatr Anaesth 2014:24:1132-40.

39. Elinder K, Söderman AC, Stalfors J, et al. Factors influencing morbidity after paediatric tonsillectomy: a study of 18,712 patients in the National Tonsil Surgery Register in Sweden. Eur Arch Otorhinolaryngol 2016;273:2249-56.

40. Bronco A, Pietrini D, Lamperti M, et al. Incidence of pain after craniotomy in children. Paediatr Anaesth 2014;24:781-7.

41. Avian A, Messerer B, Wünsch G, et al. Postoperative paediatric pain prevalence: a retrospective analysis in a university teaching hospital. Int J Nurs Stud 2016;62:36-43.

42. Sowder JC, Gale CM, Henrichsen JL, et al. Primary caregiver perception of pain control following pediatric adenotonsillectomy: a cross-sectional survey. Otolaryngol Head Neck Surg 2016;155:869-75.

43. Berde CB, Lehn BM, Yee JD, et al. Patient-controlled analgesia in children and adolescents: a randomized, prospective comparison with intramuscular administration of morphine for postoperative analgesia. J Pediatr 1991;118:460-6.

44. Veneziano G, Martin DP, Beltran R, et al. Dexamethasone as an Adjuvant to Femoral Nerve Block in Children and Adolescents Undergoing Knee Arthroscopy: a Prospective, Randomized, Double-Blind, Placebo-Controlled Trial. Reg Anesth Pain Med 2018;43:438-44

45. Alcântara CE, Falci SG, Oliveira-Ferreira F, et al. Pre-emptive effect of dexamethasone and methylprednisolone on pain, swelling, and trismus after third molar surgery: a split-mouth randomized tripleblind clinical trial. Int J Oral Maxillofac Surg 2014;43:93-8.

46. Moher D, Hopewell S, Schulz KF, et al. CONSORT 2010 explanation and elaboration: updated guidelines for reporting parallel group randomised trials. BMJ 2010;340:c869.

47. International Committee of Medical Journal Editors (ICMJE). International Committee of Medical Journal Editors (ICMJE): uniform requirements for manuscripts submitted to Biomedical Journals: writing and editing for biomedical publication. Haematologica 2004;89:264. 